\title{
Mean Arterial Blood Pressure Monitoring Among Pediatric Patients after Open Heart Surgery
}

\author{
Hanaa Hussein El-Sayed Ahmed; PhD ${ }^{(1)}$, Suad El-Sayed Abdel-Motaleb El- \\ Saman; PhD ${ }^{(2)}$, Belal Ibrahim Mohammed Essa; B.sc, R. N. ${ }^{(3)}$ \\ ${ }^{(1)}$ Department of Critical Care and Emergency Nursing, Faculty of Nursing, Mansoura University, Egypt. \\ ${ }^{(2)}$ Department of Critical Care and Emergency Nursing, Faculty of Nursing, Alexandria University, Egypt \\ (3) Data Collector, Cardiothoracic Intensive Care Unit, Children Hospital, Alexandria University, Egypt
}

\begin{abstract}
Background: Children undergoing open heart surgery may have quick and considerable changes in mean arterial pressure reading that may lead to decreased tissue perfusion. Mean arterial pressure monitoring pointed to rectifying deviations before the consequences of either hypertension or hypotension are recorded. The bedside measurement of continued mean arterial pressure reading has been routinely obtainable noninvasively or invasively.

Aim: to assess mean arterial pressure monitoring among pediatric patients after open heart surgery.

Design: A descriptive study was used.

Setting: the study was achieved at cardiac surgery intensive care unit of Children Hospital, Mansoura University, Egypt.

Subjects: A convenience sample of 50 child patients of both sexes undergoing open heart surgery aged in between 1 to 6 years old.

Tool: "Mean arterial blood pressure record" tool was used.

Results: in the studied patients, nearly two thirds of them were males (68\%) and mean age was $2.39 \pm 1.57$ years. There was no significant difference between invasive and noninvasive mean arterial blood pressure readings at preoperative and all over postoperative 12 hours but there was a highly significant correlation between them. There was no significant correlation between noninvasive and invasive postoperative MAP readings with vasoactive drug doses of Dopamine and Dobutamine but there was a significant correlation between non-invasive and invasive postoperative mean arterial blood pressure readings with vasoactive drug doses of Epinephrine in postoperative 5th, 9th, 10th, 11th and 12th hour.

Conclusions: the present study confirmed that the changes in mean arterial blood pressure measurements by noninvasive method were reflected similarly to those by invasive method.

Recommendations: critical care nurses can depend on noninvasive method alternatively to invasive one for monitoring mean arterial blood pressure. Further research is needed to study heart rate changes after cardiac surgery in pediatric patients.
\end{abstract}

Keywords: mean arterial pressure, open heart surgery, monitoring, noninvasive, invasive, critical care nurse

\section{Introduction}

Critical care nurses have a crucial role in caring for pediatric patients after open heart surgery. Their responsibilities focus different aspects as monitoring cardiovascular alterations and response to therapy postoperatively. Postoperative care of the cardiac surgical pediatric patients has the same value of surgery itself. So, pediatric patient's postoperatively clinical evaluation has to be complete and systematic. ${ }^{(1)}$ Adequate standardized monitoring postoperatively folds a clinical evaluation and the relationship between systemic and pulmonary blood flow. Sophisticated standard monitoring may be added to facilitate clinical diagnosis and treatment that consists of ECG, temperature probe, central venous pressure and arterial blood pressure (ABP). ${ }^{(2)}$ ABP folds main three components as follow systolic, diastolic and mean arterial pressure (MAP). MAP is the mean pressure averaged over time in the arterial pressure readings. ${ }^{(3,4)}$

Pediatric patients undergoing open heart surgery may have quick and considerable changes in MAP reading that may lead to decreased tissue perfusion. MAP monitoring pointed to rectifying deviations before the consequences of either hypertension or hypotension are recorded ${ }^{(3)} \mathrm{ABP}$ is a frequent clinical procedure that can be measured noninvasively or invasively. ${ }^{(5)}$ Noninvasive MAP reading using the automatic oscillometric technique with an upper arm inflatable cuff is the most used method postoperatively. ${ }^{(6)}$ This method is the easiest, relatively fast, and cheapest one; but intermittent technique. Thereby, this method is less suitable for monitoring patients with very fast changes in MAP and may be less reliable compared with invasive MAP reading. ${ }^{(7,8)}$ 
Invasive MAP is more reliable and continued arterial pressure reading. The most common indication for invasive MAP monitoring is for continuous evaluation for hemodynamically unstable pediatric pateints. ${ }^{(9)}$ The bedside measurement of continued MAP reading has been routinely obtainable through arterial catheterization. (3) Anywise, arterial catheter placement in children is unfavorable before anesthesia administration. Radial artery is the most frequently used, while the femoral artery is the second choice. (9) Invasive MAP monitoring has been used in critically ill pediatric patients, in both the operating room and intensive care units. It simplifies the immediate diagnoses of cardiovascular insufficiency and monitor therapy outcome. ${ }^{(3)}$ Thence, it can be a time devour, and necessitates a spacious experience. Over and above, this method is inherent with a risk of bleeding, infection, and distal limb ischemia for these reasons; noninvasive MAP is until now repeatedly used in very young pediatric patients. ${ }^{(7,10)}$ Invasive MAP monitoring is a widespread procedure in critical care units. Its reliability is pivotal in assessing the circulatory system and managing medication therapy for hemodynamic support. Scarcity of conclusive information in critically ill pediatric patients creates many difficulties to establish guidelines for routine practice in critical care settings. ${ }^{(9)}$ The requisition support for sufficient tissue perfusion and oxygen transport is essentially associated with inotropic and vasodilator agents administration. Inotropic agents are the fundamental curative tools for curing cardiovascular insufficiencies. The fundamental sympathomimetic vasoactive agents are illustrated by Dopamine, Dobutamine and Epinephrine. Dopamine accelerates myocardial contractility via multiple mechanisms involving stimulation of postsynaptic beta 1 -adrenergic receptors. While dobutamine rises cardiac output and minimizes systemic vascular resistance and ventricular filling pressure. Otherwise, Epinephrine is an endogenous catecholamine that is produced from the adrenal medulla and released from Norepinephrine. Its action affects alpha, beta 1 - and 2-adrenergic receptors. ${ }^{(11)}$ Depending on its dose, it raises heart rate and systolic arterial pressure, declines diastolic arterial pressure, and relaxes the peripheral vascular bed. ${ }^{(2,12)}$ The reliability of noninvasive MAP compared with invasive MAP reading has been monitored by many authors in adult patients undergoing cardiac surgery. ${ }^{(9,13-15)}$

Unluckily, the pediatric patients undergoing cardiac surgery are still a big issue for investigation and monitoring due to the vigorous risks and its difficulties. From the above the critical care nurses have a dynamic role in monitoring and recording cardiovascular alterations especially MAP to ensure child's well-being after open heart surgery. They have to closely monitor pediatric patient's ABP as MAP postoperatively to evaluate any deviations among them. Thus, our research planned to monitor MAP among pediatric patients after open heart surgery throughout twelve hours postoperatively.

\section{Aim of the study}

To assess mean arterial blood pressure monitoring among pediatric patients after open heart surgery.

\section{Research hypothesis}

- Mean arterial blood pressure reading by noninvasive method is reliable as invasive among pediatric patients after open heart surgery.

\section{Research design}

\section{Materials and Method}

A descriptive design was used in carrying out this study.

Setting

The research was carried out at Cardiac Surgery Intensive Care Unit of Children Hospital, Mansoura University, Egypt.

\section{Patients}

A convenience sample of 50 pediatric patients was selected on the study. Patients were included according to the following inclusion criteria; patients with both gender, aged in between 1 to 6 years old, and undergoing open heart surgery during immediate preoperative and twelve hours postoperative, connected to radial arterial catheter for invasive mean arterial blood pressure monitoring. Pediatric patients with hemodynamic instability and bleeding tendency were excluded from the study.

Tool:

A utilized tool was "Mean arterial blood pressure record". This tool was developed by the researchers after reviewing the related literature ${ }^{(16-22)}$. The tool was utilized to assess definite items related to MAP. The tool was divided into two parts:

Part I: Patient's profile sheet; it involved patients' gender, age, diagnosis, anthropometric measurements [weight, height], vital signs [heart rate (HR), respiratory rate (RR) and temperature (Temp)], blood studies [hematocrit (Hct), platelets (Plts), prothrombine time (PT), INR, creatinine] that were taken preoperatively.

Part II: Noninvasive and invasive mean arterial blood pressure record; it consisted of invasive and noninvasive mean arterial blood pressure, and vasoactive agents' name and its dose each hour throughout 12 hours postoperatively. 
Tool preparation and validity:

The tool was developed by the researchers based on reviewing the related literature. The content validity of the tool was reviewed by 5 experts (two critical care nursing professors, two cardiothoracic surgeons, and one cardiologist). The required changes were done according to their knowledge and clinical experience.

Pilot study

A pilot study was carried out on five patients after obtaining family's pediatric patients' consents who were excluded from the research subjects in order to assess the clarity and the applicability of the tool. The required changes were done accordingly.

\section{Procedure}

Pediatric patients were selected according to the criteria. Redial arterial catheter was inserted to studied patients. For all studied patient, noninvasive MAP was monitored by automatic oscillometric method once at immediate preoperative and hourly for twelve postoperative hours. Additionally, for all studied patient, invasive MAP was monitored from radial catheter once at immediate preoperative and hourly for twelve postoperative hours. The obtained MAP readings were recorded using part II of the tool. Administered vasoactive drugs name and doses were recorded hourly for twelve postoperative hours for the studied patients. To assess MAP monitoring among pediatric patients after open heart surgery comparison between noninvasive and invasive MAP reading was done. Data collection took approximately six months from September 2015 to March 2016.

\section{Administrative design and ethical considerations}

Approval permission to conduct the research was obtained from the hospital administrative personnel based on the official letter after explanation the aim and nature of the research. The present research was approved by the Scientific Research Ethics Committee of the Faculty of Nursing- Mansoura University. Informed consents were obtained from the pediatric patients' family before the beginning of the research after explanation of the research purpose. Confidentiality of the collected data was assured and participants were able to withdraw from the study at any stage. Data collection took approximately six months from September 2015 to March 2016.

\section{Statistical analysis of the data}

Data were fed to the computer and analyzed using IBM SPSS software package version 20. Qualitative data were described using number and percent. Quantitative data were described using minimum and maximum, mean and standard deviation. Comparisons were done using: Pearson coefficient (r), and $\mathrm{p}$ value for value for Psost Hoc test (LSD) for ANOVA with repeated measures for comparing between preoperative and vasoactive agents period. $\mathrm{P}$ is significant if $\leq 0.05$.

\section{Results}

Table 1 shows distribution of the studied patients according to their profile data. It was noticed in the studied patients that nearly two thirds of them were males $(68 \%)$. Mean age was $2.39 \pm 1.57$ years. Mean body weight was $11.4 \pm 5.15 \mathrm{~kg}$. Mean heart rate was $125.86 \pm 15.28 \mathrm{~b} / \mathrm{min}$. Mean respiratory rate was $40.18 \pm 11.01 \mathrm{c} / \mathrm{min}$. Mean temperature was $36.83 \pm 0.82^{\circ} \mathrm{C}$.

Table 2 illustrates comparison between patients' preoperative and postoperative first 12 hours mean arterial blood pressure (MAP) reading. The table revealed that there was a highly significant difference between invasive and non-invasive MAP readings through 11 postoperative hours with preoperative baseline reading. However, in 12th postoperative hour, this significant difference disappeared with non-invasive $(p=0.228)$ and invasive ( $\mathrm{p}=0.394)$.

Table 3 shows comparison between invasive and noninvasive MAP readings at preoperative and postoperative first 12 hours. The results showed no significant difference between them all over 12 postoperative hours.

Table 4 shows correlation between noninvasive and invasive MAP readings at preoperative and postoperative first 12 hours. The results showed highly significant correlation between them all over the 12 postoperative hours.

Table 5 shows correlation between patients' postoperative first 12 hours MAP readings with administered vasoactive drugs doses. The table illustrated that there was no significant correlation between noninvasive and invasive postoperative MAP readings with vasoactive drug doses of Dopamine and Dobutamine. On the other hand, there was a significant correlation between non-invasive and invasive postoperative MAP readings with vasoactive drug doses of Epinephrine in postoperative 5th, 9th, 10th, 11th and 12th hour. (For non-invasive: $0.050,0.012,0.001,0.045$ and 0.005 respectively and for invasive: $0.040,0.022,0.009,0.055$ and 0.026 respectively).

Table 6 demonstrates correlation between patients' profile data and MAP readings through first 12 hours postoperatively. It was noticed that there was no significant correlation between them. 
Mean arterial blood pressure monitoring among pediatric patients after open heart surgery

Table 1: Distribution of the studied patients according to their profile data

\begin{tabular}{|c|c|c|c|c|c|}
\hline \multicolumn{2}{|r|}{ Items } & \multicolumn{4}{|c|}{ The studied patients $(n=50)$} \\
\hline \multirow[b]{2}{*}{ Gender } & \multirow[b]{2}{*}{$\begin{array}{l}\text { Male } \\
\text { Female }\end{array}$} & \multicolumn{2}{|c|}{ No } & \multicolumn{2}{|c|}{$\%$} \\
\hline & & \multicolumn{2}{|c|}{$\begin{array}{l}34 \\
16 \\
\end{array}$} & \multicolumn{2}{|c|}{$\begin{array}{l}68 \\
32 \\
\end{array}$} \\
\hline & & Min & Max & Mean & $\pm \mathrm{SD}$ \\
\hline Age (years & & 1.0 & 6.0 & 2.39 & 1.57 \\
\hline $\begin{array}{l}\text { Anthropometric } \\
\text { measurements }\end{array}$ & $\begin{array}{l}\text { Weight }(\mathrm{kg}) \\
\text { Height }(\mathrm{cm})\end{array}$ & $\begin{array}{l}4.25 \\
28.0\end{array}$ & $\begin{array}{c}27.0 \\
129.0\end{array}$ & $\begin{array}{l}11.44 \\
79.82\end{array}$ & $\begin{array}{c}5.15 \\
19.14 \\
\end{array}$ \\
\hline Vital signs & $\begin{array}{l}\text { Heart Rate } \\
\text { Respiratory Rate } \\
\text { Temperature }\left({ }^{\circ} \mathrm{C}\right)\end{array}$ & $\begin{array}{l}83.0 \\
30.0 \\
36.0\end{array}$ & $\begin{array}{c}160.0 \\
110.0 \\
42.0 \\
\end{array}$ & $\begin{array}{c}125.86 \\
40.18 \\
36.83 \\
\end{array}$ & $\begin{array}{c}15.28 \\
11.01 \\
0.82\end{array}$ \\
\hline $\begin{array}{c}\text { Blood chemistry } \\
\text { studies }\end{array}$ & $\begin{array}{l}\text { Hematocrit } \\
\text { Platelets } \\
\text { Prothrombine time } \\
\text { INR } \\
\text { Creatinine }\end{array}$ & $\begin{array}{c}29.40 \\
79.0 \\
12.0 \\
1.0 \\
0.20\end{array}$ & $\begin{array}{c}63.20 \\
485.0 \\
17.57 \\
1.90 \\
0.70\end{array}$ & $\begin{array}{c}39.57 \\
292.85 \\
12.76 \\
1.04 \\
0.45\end{array}$ & $\begin{array}{c}8.28 \\
96.98 \\
1.17 \\
0.14 \\
0.12\end{array}$ \\
\hline
\end{tabular}

Table 2: Comparison between patients' preoperative baseline and postoperative first 12 hours mean arterial blood pressure readings.

\begin{tabular}{|c|c|c|c|c|c|c|c|c|c|c|c|}
\hline \multirow{3}{*}{\multicolumn{2}{|c|}{ Hours }} & \multicolumn{10}{|c|}{ Mean arterial blood pressure $(n=50)$} \\
\hline & & \multicolumn{5}{|c|}{ Noninvasive } & \multicolumn{5}{|c|}{ Invasive } \\
\hline & & Min & Max & Mean & \pm SD & ${ }^{\text {LSD }} \mathbf{p}$ & Min & Max & Mean & \pm SD & ${ }^{\mathrm{LSD}} \mathbf{p}$ \\
\hline \multicolumn{2}{|c|}{ Preoperative } & 54.0 & 118.0 & 76.6 & 13.0 & & 57.0 & 110.0 & 78.4 & 12.0 & \\
\hline \multirow{12}{*}{ 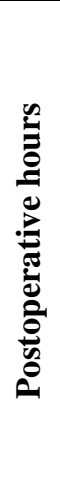 } & $1^{\text {st }}$ & $67.0-$ & 135.0 & 86.6 & 13.4 & $0.001^{*}$ & 80.0 & 120.0 & 86.1 & 17.2 & $0.008^{*}$ \\
\hline & $2^{\text {nd }}$ & 61.0 & 130.0 & 85.3 & 14.0 & $0.001^{*}$ & 62.0 & 122.0 & 87.8 & 12.9 & $0.001^{*}$ \\
\hline & $3^{\text {rd }}$ & 65.0 & 119.0 & 85.6 & 13.1 & $0.001^{*}$ & 57.0 & 122.0 & 85.7 & 12.8 & $0.004^{*}$ \\
\hline & $4^{\text {th }}$ & 62.0 & 113.0 & 86.1 & 13.0 & $0.001^{*}$ & 59.0 & 115.0 & 85.7 & 12.85 & $0.008^{*}$ \\
\hline & $5^{\text {th }}$ & 58.0 & 108.0 & 84.3 & 12.7 & $0.002^{*}$ & 62.0 & 113.0 & 84.9 & 12.4 & $0.010^{*}$ \\
\hline & $6^{\text {th }}$ & 52.0 & 110.0 & 82.7 & 11.2 & $0.012^{*}$ & 60.0 & 111.0 & 85.1 & 12.4 & $0.006^{*}$ \\
\hline & $7^{\text {th }}$ & 60.0 & 113.0 & 85.8 & 12.2 & $0.001^{*}$ & 62.0 & 109.0 & 85.7 & 11.2 & $0.002^{*}$ \\
\hline & $8^{\text {th }}$ & 64.0 & 105.0 & 84.9 & 11.2 & $0.001^{*}$ & 57.0 & 106.0 & 83.3 & 12.0 & $0.027^{*}$ \\
\hline & $9^{\text {th }}$ & 50.0 & 117.0 & 85.7 & 13.7 & $0.002^{*}$ & 62.0 & 122.0 & 84.2 & 12.3 & $0.030^{*}$ \\
\hline & $10^{\text {th }}$ & 56.0 & 111.0 & 84.7 & 12.9 & $0.002^{*}$ & 57.0 & 110.0 & 83.9 & 11.1 & $0.008^{*}$ \\
\hline & $11^{\text {th }}$ & 47.0 & 113.0 & 84.6 & 14.1 & $0.003^{*}$ & 60.0 & 114.0 & 85.0 & 12.4 & $0.004^{*}$ \\
\hline & $12^{\text {th }}$ & 49.0 & 116.0 & 80.1 & 14.7 & 0.228 & 51.0 & 117.0 & 80.5 & 12.9 & 0.394 \\
\hline
\end{tabular}

$\mathrm{p}$ : $\mathrm{p}$ value for value of Post Hoc test (LSD) for ANOVA with repeated *: Statistically significant at $\mathrm{p} \leq 0.05$

Table 3: Comparison between non-invasive and invasive mean arterial blood pressure reading at preoperative and postoperative first 12 hours.

\begin{tabular}{|c|c|c|c|c|c|c|c|}
\hline \multirow{3}{*}{\multicolumn{2}{|c|}{$\begin{array}{c}\text { Postoperative } \\
\text { hours }\end{array}$}} & \multicolumn{4}{|c|}{ Mean arterial blood pressure $(\mathrm{N}=50)$} & \multirow{3}{*}{$\mathbf{T}$} & \multirow{3}{*}{$\mathbf{P}$} \\
\hline & & \multicolumn{2}{|c|}{ Non-invasive } & \multicolumn{2}{|c|}{ invasive } & & \\
\hline & & Mean & \pm SD & Mean & $\pm \mathrm{SD}$ & & \\
\hline $\operatorname{Pr}$ & tive & 76.6 & 13.0 & 78.4 & 12.0 & 0.742 & 0.802 \\
\hline \multirow{12}{*}{ 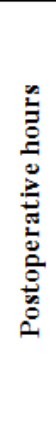 } & $1^{\text {st }}$ & 86.6 & 13.4 & 86.1 & 17.2 & 0.248 & 0.805 \\
\hline & $2^{\text {nd }}$ & 85.3 & 14.0 & 87.8 & 12.9 & 1.855 & 0.070 \\
\hline & $3^{\text {th }}$ & 85.6 & 13.1 & 85.7 & 12.8 & 0.110 & 0.901 \\
\hline & $4^{\text {th }}$ & 86.1 & 13.0 & 85.7 & 12.85 & 0.329 & 0.744 \\
\hline & $5^{\text {th }}$ & 84.3 & 12.7 & 84.9 & 12.4 & 0.438 & 0.663 \\
\hline & $6^{\text {th }}$ & 82.7 & 11.2 & 85.1 & 12.4 & 1.766 & 0.084 \\
\hline & $7^{\text {th }}$ & 85.8 & 12.2 & 85.7 & 11.2 & 0.111 & 0.912 \\
\hline & $8^{\text {th }}$ & 84.9 & 11.2 & 83.3 & 12.0 & 1.400 & 0.168 \\
\hline & $9^{\text {th }}$ & 85.7 & 13.7 & 84.2 & 12.3 & 0.874 & 0.386 \\
\hline & $10^{\text {th }}$ & 84.7 & 12.9 & 83.9 & 11.1 & 0.756 & 0.453 \\
\hline & $11^{\text {th }}$ & 84.6 & 14.1 & 85.0 & 12.4 & 0.361 & 0.719 \\
\hline & $12^{\text {th }}$ & 80.1 & 14.7 & 80.5 & 12.9 & 0.697 & 0.489 \\
\hline
\end{tabular}

Test used: T-test

*: Statistically significant at $\mathrm{p} \leq 0.05$ 
Table 4: Correlation between noninvasive and invasive mean blood pressure readings at preoperative and postoperative first 12 hours.

\begin{tabular}{|c|c|c|c|}
\hline \multirow{2}{*}{\multicolumn{2}{|c|}{ Invasive blood pressure }} & \multicolumn{2}{|c|}{ Non Invasive blood pressure } \\
\hline & & $\mathbf{r}$ & $\mathbf{P}$ \\
\hline \multicolumn{2}{|c|}{ Preoperative } & $0.676^{*}$ & $<0.001^{*}$ \\
\hline \multirow{12}{*}{ 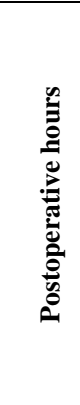 } & $1^{\text {st }}$ & $0.517^{*}$ & $<0.001^{*}$ \\
\hline & $2^{\text {nd }}$ & $0.753^{*}$ & $<0.001^{*}$ \\
\hline & $3^{\text {th }}$ & $0.756^{*}$ & $<0.001^{*}$ \\
\hline & $4^{\text {th }}$ & $0.800^{*}$ & $<0.001^{*}$ \\
\hline & $5^{\text {th }}$ & $0.680^{*}$ & $<0.001^{*}$ \\
\hline & $6^{\text {th }}$ & $0.672^{*}$ & $<0.001^{*}$ \\
\hline & $7^{\text {th }}$ & $0.791^{*}$ & $<0.001^{*}$ \\
\hline & $8^{\text {th }}$ & $0.771^{*}$ & $<0.001^{*}$ \\
\hline & $9^{\text {th }}$ & $0.520^{*}$ & $<0.001^{*}$ \\
\hline & $\mathbf{1 0}^{\text {th }}$ & $0.845^{*}$ & $<0.001^{*}$ \\
\hline & $11^{\text {th }}$ & $0.833^{*}$ & $<0.001^{*}$ \\
\hline & $12^{\text {th }}$ & $0.863^{*}$ & $<0.001^{*}$ \\
\hline \multicolumn{2}{|c|}{ Average blood pressure } & $0.856^{*}$ & $<0.001^{*}$ \\
\hline
\end{tabular}

$$
\text { r: Pearson coefficient } \quad \text { *: Statistically significant at } \mathrm{p} \leq 0.05
$$

Table 5: Correlation between patients' postoperative first 12 hours mean arterial blood pressure readings with administered vasoactive drugs doses.

\begin{tabular}{|c|c|c|c|c|c|}
\hline \multirow{3}{*}{$\begin{array}{l}\text { Vasoactive } \\
\text { drugs }\end{array}$} & \multirow{3}{*}{ Hours } & \multicolumn{4}{|c|}{ Mean arterial blood pressure $(n=50)$} \\
\hline & & \multicolumn{2}{|c|}{ Noninvasive } & \multicolumn{2}{|c|}{ Invasive } \\
\hline & & $\mathbf{r}$ & $\mathbf{P}$ & $\mathbf{R}$ & $\mathbf{P}$ \\
\hline 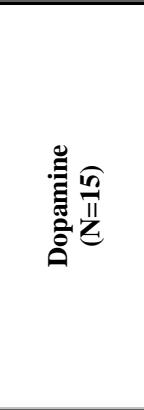 & $\begin{array}{c}1^{\text {st }} \\
2^{\text {nd }} \\
3^{\text {rd }} \\
4^{\text {th }} \\
5^{\text {th }} \\
6^{\text {th }} \\
7^{\text {th }} \\
8^{\text {th }} \\
9^{\text {th }} \\
10^{\text {th }} \\
11^{\text {th }} \\
12^{\text {th }}\end{array}$ & $\begin{array}{c}-0.195 \\
-0.170 \\
-0.160 \\
-0.211 \\
-0.045 \\
0.006 \\
-0.171 \\
-0.185 \\
-0.149 \\
-0.151 \\
-0.048 \\
-0.157\end{array}$ & $\begin{array}{l}0.174 \\
0.237 \\
0.268 \\
0.141 \\
0.755 \\
0.967 \\
0.236 \\
0.199 \\
0.303 \\
0.296 \\
0.739 \\
0.276\end{array}$ & $\begin{array}{c}-0.018 \\
-0.121 \\
-0.133 \\
-0.149 \\
0.050 \\
-0.040 \\
-0.126 \\
-0.136 \\
0.100 \\
-0.108 \\
-0.057 \\
-0.093\end{array}$ & $\begin{array}{l}0.903 \\
0.401 \\
0.359 \\
0.301 \\
0.732 \\
0.785 \\
0.382 \\
0.347 \\
0.488 \\
0.455 \\
0.695 \\
0.521\end{array}$ \\
\hline 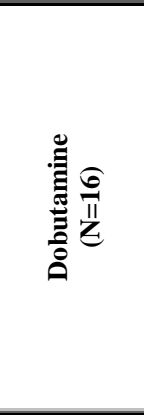 & $\begin{array}{c}1^{\text {st }} \\
2^{\text {nd }} \\
3^{\text {rd }} \\
4^{\text {th }} \\
5^{\text {th }} \\
6^{\text {th }} \\
7^{\text {th }} \\
8^{\text {th }} \\
9^{\text {th }} \\
10^{\text {th }} \\
11^{\text {th }} \\
12^{\text {th }}\end{array}$ & $\begin{array}{c}0.145 \\
0.220 \\
0.070 \\
0.240 \\
0.082 \\
0.099 \\
0.222 \\
0.144 \\
0.007 \\
0.009 \\
-0.167 \\
-0.032\end{array}$ & $\begin{array}{l}0.316 \\
0.124 \\
0.630 \\
0.093 \\
0.570 \\
0.494 \\
0.121 \\
0.319 \\
0.963 \\
0.948 \\
0.245 \\
0.823\end{array}$ & $\begin{array}{c}-0.126 \\
-0.006 \\
-0.123 \\
0.174 \\
-0.094 \\
-0.092 \\
0.089 \\
-0.088 \\
-0.032 \\
-0.092 \\
-0.180 \\
-0.197 \\
\end{array}$ & $\begin{array}{l}0.382 \\
0.965 \\
0.394 \\
0.227 \\
0.517 \\
0.526 \\
0.538 \\
0.545 \\
0.825 \\
0.524 \\
0.212 \\
0.170 \\
\end{array}$ \\
\hline 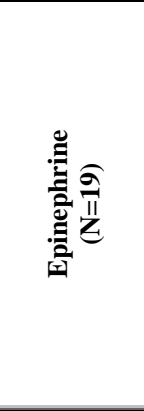 & $\begin{array}{c}1^{\text {st }} \\
2^{\text {nd }} \\
3^{\text {rd }} \\
4^{\text {th }} \\
5^{\text {th }} \\
6^{\text {th }} \\
7^{\text {th }} \\
8^{\text {th }} \\
9^{\text {th }} \\
10^{\text {th }} \\
11^{\text {th }} \\
12^{\text {th }}\end{array}$ & $\begin{array}{l}-0.010 \\
-0.195 \\
-0.052 \\
-0.239 \\
-0.279 \\
-0.208 \\
-0.223 \\
-0.293 \\
-0.352 \\
-0.438 \\
-0.284 \\
-0.392\end{array}$ & $\begin{array}{c}0.944 \\
0.174 \\
0.718 \\
0.095 \\
0.050^{*} \\
0.147 \\
0.119 \\
0.039^{*} \\
0.012^{*} \\
0.001^{*} \\
0.045^{*} \\
0.005^{*}\end{array}$ & $\begin{array}{c}0.004 \\
-0.246 \\
-0.090 \\
-0.369 \\
-0.292 \\
-0.278 \\
-0.161 \\
-0.200 \\
-0.324 \\
-0.366 \\
-0.273 \\
-0.314 \\
\end{array}$ & $\begin{array}{c}0.976 \\
0.085 \\
0.532 \\
0.008^{*} \\
0.040^{*} \\
0.051^{*} \\
0.263 \\
0.163 \\
0.022^{*} \\
0.009^{*} \\
0.055^{*} \\
0.026^{*}\end{array}$ \\
\hline
\end{tabular}

r: Pearson coefficient $\quad *$ : Statistically significant at $\mathrm{p} \leq 0.05$ 
Mean arterial blood pressure monitoring among pediatric patients after open heart surgery

Table 6: Correlation between patients' profile data and mean arterial blood pressure readings through first 12 hours postoperatively

\begin{tabular}{|c|c|c|c|c|c|c|c|c|c|c|c|c|c|}
\hline \multirow{4}{*}{\multicolumn{2}{|c|}{ Hours }} & \multicolumn{12}{|c|}{ Mean arterial blood pressure $(n=50)$} \\
\hline & & \multicolumn{6}{|c|}{ Noninvasive } & \multicolumn{6}{|c|}{ Invasive } \\
\hline & & \multicolumn{2}{|c|}{ Age } & \multicolumn{2}{|c|}{ Weight } & \multicolumn{2}{|c|}{ Height } & \multicolumn{2}{|c|}{ Age } & \multicolumn{2}{|c|}{ Weight } & \multicolumn{2}{|c|}{ Height } \\
\hline & & $\mathbf{R}$ & $\mathbf{P}$ & $\mathbf{r}$ & $\mathbf{P}$ & $\mathbf{r}$ & $\mathbf{p}$ & $\mathbf{r}$ & $\mathbf{P}$ & $\mathbf{r}$ & $\mathbf{p}$ & $\mathbf{r}$ & $\mathbf{p}$ \\
\hline \multirow{12}{*}{ 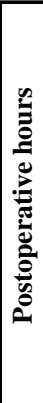 } & $1^{\text {st }}$ & 0.110 & 0.448 & 0.097 & 0.505 & 0.193 & 0.180 & 0.132 & 0.360 & 0.076 & 0.601 & 0.046 & 0.749 \\
\hline & $2^{\text {nd }}$ & 0.124 & 0.390 & 0.108 & 0.457 & 0.121 & 0.404 & 0.113 & 0.434 & 0.065 & 0.655 & 0.081 & 0.574 \\
\hline & $3^{\text {th }}$ & 0.081 & 0.578 & 0.068 & 0.641 & 0.047 & 0.747 & 0.002 & 0.991 & -0.026 & 0.859 & -0.054 & 0.707 \\
\hline & $4^{\text {th }}$ & -0.015 & 0.918 & -0.010 & 0.946 & -0.089 & 0.540 & -0.027 & 0.851 & -0.047 & 0.748 & -0.054 & 0.712 \\
\hline & $5^{\text {th }}$ & -0.014 & 0.923 & -0.033 & 0.822 & 0.032 & 0.824 & 0.021 & 0.885 & -0.084 & 0.564 & -0.06 & 0.678 \\
\hline & $6^{\text {th }}$ & 0.024 & 0.870 & -0.015 & 0.917 & 0.033 & 0.819 & 0.01 & 0.946 & -0.029 & 0.841 & 0.041 & 0.778 \\
\hline & $7^{\text {th }}$ & 0.042 & 0.772 & 0.052 & 0.720 & 0.087 & 0.548 & -0.011 & 0.938 & -0.037 & 0.800 & -0.023 & 0.873 \\
\hline & $8^{\text {th }}$ & 0.099 & 0.494 & 0.096 & 0.505 & 0.170 & 0.238 & 0.172 & 0.233 & 0.132 & 0.361 & 0.151 & 0.294 \\
\hline & $9^{\text {th }}$ & -0.128 & 0.377 & -0.157 & 0.276 & -0.095 & 0.513 & -0.02 & 0.890 & -0.022 & 0.880 & -0.031 & 0.830 \\
\hline & $10^{\text {th }}$ & 0.051 & 0.728 & 0.009 & 0.950 & 0.015 & 0.919 & 0.108 & 0.455 & 0.066 & 0.648 & 0.007 & 0.963 \\
\hline & $11^{\text {th }}$ & 0.040 & 0.783 & -0.035 & 0.810 & -0.045 & 0.756 & 0.08 & 0.579 & -0.006 & 0.966 & 0.035 & 0.807 \\
\hline & $12^{\text {th }}$ & 0.034 & 0.815 & -0.125 & 0.386 & 0.046 & 0.750 & 0.112 & 0.438 & -0.083 & 0.567 & 0.056 & 0.698 \\
\hline
\end{tabular}

r: Pearson coefficient

*: Statistically significant at $\mathrm{p} \leq 0.05$

\section{Discussion}

This study concentrated on mean arterial blood pressure (MAP) in pediatric patients. This is due to that MAP is preferred for valuing the flow of peripheral blood. Moreover, pediatric patients are a high-risk group so it is valued to discuss issues related to them. ${ }^{(23)}$ Lehman et al. ${ }^{(24)}$ suggested that MAP is a favored metrical guide in intensive care units. Moreover, Balzer et al ${ }^{(25)}$ stated that assessing arterial blood pressure (ABP) in postoperative stage is a central principle of medical staff alertness and ABP interventions is an essential focus of their postoperative care. Therefore, monitoring ABP should be supported by evidence base. ${ }^{(26)}$

From the current study, it was found that there was a highly significant difference between invasive or noninvasive MAP readings during 11 postoperative hours with preoperative baseline reading and this significant difference disappeared at $12^{\text {th }}$ postoperative hour. It means that in the studied pediatric patients, MAP returned near to its preoperative baseline after about 12 hours. It can be attributed to that hemodynamically stability in children is affected by intraoperative blood loss than adults. ${ }^{(27)}$ Demirci et al. ${ }^{(28)}$ approved that hemodynamical instability was common to happen post-operatively in cardiac surgical patients while waiting for reaching stability by vasoactive drugs support in intensive care unit. Additionally, these patients need close monitoring and recording of vital signs hourly. This interval was based on cardiac surgical intensive care nurses' experience.

Additionally, these results were augmented by Nikolov et al. ${ }^{(29)}$ who approved that cardiac patients are exposed to tensions of surgical interventions, especially patients with pre-existing risk factors as age of pediatric patients; these patients are subjected to high fluctuation in post-operative BP value that differs greatly from preoperative baseline. Equally, Mangano ${ }^{(30)}$ and Breisblatt et al. ${ }^{(31)}$ showed decreased postoperative heart function in relation to preoperative in patients with cardiac surgery.

The current results demonstrated that there was no significant difference between invasive and noninvasive MAP readings through postoperative12 hours and there was a highly significant correlation between them. This means that there was closeness between non-invasive and invasive MAP readings for these paediatric patients so critical care nurses (CCNs) can depend on using non-invasive method to obtain accurate MAP reading alternatively to invasive method. In the same time generally, using non-invasive method is simpler, save, less complications as bleeding and distal limb ischemia and less cost than invasive method. ${ }^{(32)}$ These results were in line with Andriessen et al. ${ }^{(33)}$ who concluded that in pediatric patients, non-invasive ABP reading showed worthy similarities with invasive ones. Additionally, Gevers et al. ${ }^{(34)}$ concluded presence of a big similarity between invasive and non-invasive ABP reading.

Lehman et al. ${ }^{(24)}$ approved that MAP from noninvasive and invasive methods can be taken in a dependable way in evaluating patient's condition. Furthermore, Hofhuizen et al. ${ }^{(35)}$ concluded that non-invasive brachial artery pressure was adequately reflects changes as invasive arterial pressure in cardiac children patients through surgical procedure. Hori et al. ${ }^{(36)}$ illustrated that hemodynamical instability though cardiac surgery was a risk reason for developing cardiac surgical complication and they depend on monitoring MAP by noninvasive method to discover these complications. Jagadeesh, et al. ${ }^{(37)}$ revealed satisfactory consistency between noninvasive reading and other reading created by invasive method in patients after cardiac surgery. On the other hand, Heard et al. ${ }^{(38)}$ disagreed with these results regarding relationship between non-invasive \& invasive as they analyzed ABP data obtained by noninvasive method and found variation in results when compared with invasive method. Furthermore, Acierno et al. ${ }^{(39)}$ Shih et al. ${ }^{(40)}$ and Almeida ${ }^{(41)}$ found a difference between the two methods. 
Based on that critical care nurses are responsible for monitoring patients' hemodynamical status and reporting any deviation from normal, they have to know the effect of certain drugs on vital signs to obtain hemodynamic stability. Moreover, maintaining stability of pediatric patients' hemodynamical status at postoperative stage necessitates interferences including medication administration as vasoactive medication. ${ }^{(27)}$ Demirci et al. ${ }^{(28)}$ did their study on analysis of BP at cardiac surgical patient postoperatively. They had a limitation of not analyzing the effect of vasoactive drugs on blood volume and pressure. Therefore, this study considered studying effect of different vasoactive drugs doses as Dobutamine, Dopamine and Epinepherine on MAP. The present study found that there was no significant correlation between non-invasive and invasive postoperative MAP reading with drug doses in pediatric patients received Dopamine or Dobutamine. On the other hand, there was a significant correlation between noninvasive or invasive postoperative MAP reading with Epinephrine doses. Therefore, these results can confirm greatly that obtained results by noninvasive method is similar to invasive ones. In the same line, Butterworth ${ }^{(42)}$ concluded that Dopamine and Dobutamine had stronger action and tended to increase heart rate than Epinephrine and so on stroke volume. Therefore, they reached near preoperative BP value than Epinephrine. Moreover, Butterworth et al. ${ }^{(43)}$ showed in their study in patients after coronary artery bypass graft the same results as Dobutamine raises heart rate higher than an equivalent dose of Epinephrine. Also, these results were augmented by DiSesa et a1. ${ }^{(44)}$ and Saloman et al. ${ }^{(45)}$ Finally, it was noticed that in the current study, there was no significant correlation between patients' age, weight and height data and MAP reading throughout 12 hours postoperatively in pediatric patients. Generally, this ' can be due to that blood pressure depends on pediatric patients' vascular system and circulating blood amount. Thus, pediatric patients' hemodynamic stability is affected rapidly by change in circulating blood volume. In a trial to explain why there was no correlation with age, weight and height in pediatric patients, Aronson ${ }^{(26)}$ discovered that presence of varied risk factors as operational duration, blood volume loss and surgical interventions were the main causes contribute to altering BP postoperatively in patients with greatly similar demographical data. Wesseling ${ }^{(46)}$ demonstrated that monitoring pediatric patients' blood pressure is a gold sign which must be measured correctly. Therefore, it is important to consider using appropriate cuff pressure for age to measure noninvasive arterial blood pressure. Moreover, Andriessen ${ }^{(33)}$ established in children that applying cuff pressure correctly seemed to be very serious when measuring ABP.

\section{Conclusion}

The current study demonstrated that there was no significant difference between invasive and noninvasive MAP readings at preoperative and allover postoperative 12 hours but there was a highly significant correlation between them at preoperative and all over the postoperative 12 hours. Furthermore, there was no significant correlation between noninvasive and invasive postoperative MAP readings with vasoactive drug doses of Dopamine and Dobutamine but there was a significant correlation between non-invasive and invasive postoperative MAP readings with vasoactive drug doses of Epinephrine in postoperative 5th, 9th, 10th, 11th and 12th hour. In addition, there was no significant correlation between patients' age, weight and height with MAP measurements by noninvasive and invasive methods. Therefore, the present study confirmed that the changes in mean arterial blood pressure measurements by noninvasive method were reflected similarly to those by invasive method.

\section{Recommendations}

Critical care nurses can depend on noninvasive method alternatively to invasive one for monitoring mean arterial blood pressure. Further study is needed to study heart rate changes after cardiac surgery in pediatric patients.

\section{References}

[1] DukeT, Stocker C, and Butt W. Monitoring children after cardiac surgery: a minimalist approach might be maximally effective. Critical Care and Resuscitation, 2004; 6: 306-310.

[2] Auler Jr., Barreto AC, Gimenez SC and Abellan DM. Pediatric cardiac postoperative care. Review of Hospital Clinical Facilities Medicine at San Paulo 2002; 57(3): 115-123.

[3] Esper S A. and Pinsky M R. Arterial waveform analysis. Best Practice \& Research Clinical Anaesthesiology 2014; 28: 363-380. www.elsevier.com/locate/bean.Contents lists available at Science Direct.

[4] Parr GV, Blackstone EH and Kirklin JW. Cardiac performance arid mortality early after intra-cardiac surgery in infants and young children. Circulation 1975; 51:867-874. Downloaded from http://circ.ahajournals.org/ by guest on July 9, 2011.

[5] Joffe R, Duff J, Guerra GG, Pugh J, and Joffe, AR. The accuracy of blood pressure measured by arterial line and non-invasive cuff in critically ill children. Critical Care, 2016; 20(1), 177

[6] Araghi A, Bander JJ and Guzman JA. Arterial blood pressure monitoring in overweight critically ill patients: invasive or noninvasive? Critical Care 2006; 10:R64 (doi:10.1186/cc4896). Available online http://ccforum.com/content/10/2/R64.

[7] Hofhuizen CM, Lemson J, Hemelaar AE, Settels JJ, Schraa O, Singh SK, van der Hoeven JG and Scheffer GJ. Continuous noninvasive finger arterial pressure monitoring reflects intra-arterial pressure changes in children undergoing cardiac surgery. British Journal of Anaesthesia 2010; 105 (4): 493-500. 
[8] Fischer MO, Avram R, Ca^ rjaliu I, Massetti M, Ge'rard JL., Hanouz JL and Fellahi JL. Non-invasive continuous arterial pressure and cardiac index monitoring with Nexfin after cardiac surgery. British Journal of Anaesthesia 2012; 109 (4): 514-21.

[9] Mignini MA, Piacentin E and Dubin A. Peripheral arterial blood pressure monitoring adequately tracks central arterial blood pressure in critically ill patients: an observational study. Critical Care 2006; 10 (2): Available online http://ccforum.com/content/10/2/R43.

[10] Andriessen P, Schraa O, Bosch-Ruis WV, Harkel DJ, Settels J, Oetomo SB and Blanco CE. Feasibility of noninvasive continuous finger arterial blood pressure measurements in very young children, aged 0-4 years. International Pediatric Research Foundation, Inc. 2008; 63 (6): 691-6

[11] Gillies M, Bellomo R, Doolan L and Buxton B. Bench-to-bedside review: Inotropic drug therapy after adult cardiac surgery - a systematic literature review. Critical Care 2005; 9 (3):266-279.

[12] Oualha M, Urien S, Spreux-Varoquaux O, Bordessoule A, D'Agostino I, Pouard P and Tréluyer JM. Pharmacokinetics, hemodynamic and metabolic effects of epinephrine to prevent post-operative low cardiac output syndrome in children. Critical Care 2014; 18:R23. Available online http://ccforum.com/content/18/1/R23.

[13] Chauhan S, Saxena N, Mehrotra S, Rao BH, Sahu M. Femoral artery pressures are more reliable than radial artery pressures on initiation of cardiopulmonary bypass. Journal of Cardiothoracic Vascular Anesthesia 2000, 14:274-276.

[14] Kanazawa M, Fukuyama H, Kinefuchi Y, Takiguchi M, Suzuki T. Relationship between aortic-to-radial arterial pressure gradient after cardiopulmonary bypass and changes in arterial elasticity. Anesthesiology 2003, 99:48-53.

[15] Manecke GR, Parimucha M, Stratmann G, Wilson WC, Roth DM, Auger WR, Kerr KM, Jamieson SW, Kapelanski DP, Mitchell MM. Deep hypothermic circulatory arrest and the femoral-to radial arterial pressure gradient. Journal of Cardiothoracic Vascular Anesthesia 2004, 18:175-179.

[16] Edmonds ZV, Mower WR, Lovato LM, and Lomeli R. "The reliability of vital sign measurements," Annals of Emergency Medicine. 2002; 39(3): 233-237.

[17] American National Standards Institute. Non-invasive sphygmomanometers — Part 2: Clinical investigation of automated measurement type. Association for the Advancement of Medical Instrumentation. 2013; Available at www.aami.org.

[18] Dind A, Short A, Ekholm J, and Holdgate A. The inaccuracy of automatic devices taking postural measurements in the emergency department. International Journal of Nursing Practice. 2011 Oct; 17(5):525-33.

[19] Jagomägi K, Talts J, Tähepõld P, and Raamat R. A comparison of differential oscillometric device with invasive mean arterial blood pressure monitoring in intensive care patients. Clinical Physiology and Functional Imaging. 2011 May; 31(3):188-92.

[20] Holt TR, Withington DE, and Mitchell E. "Which pressure to believe? A comparison of direct arterial with indirect blood pressure measurement techniques in the pediatric intensive care unit," Pediatric Critical Care Medicine, 2011; 12(6): 391-394.

[21] Takci S, Yigit S, Korkmaz A, and Yurdakök M. "Comparison between oscillometric and invasive blood pressure measurements in critically ill premature infants," Acta Paediatrica, International Journal of Pediatrics, 2012; 101(2): 132-135.

[22] Ribezzo S, Spina E, Di Bartolomeo S, and Sanson G. Noninvasive techniques for blood pressure measurement are not a reliable alternative to direct measurement: a randomized crossover trial in ICU. The Scientific World Journal, 2014; (2014) Article ID 353628, 8 pages. http://dx.doi.org/10.1155/2014/353628.

[23] Marino P and Sutin K. The ICU Book. Philadelphia, PA: Lippincott, Williams \& Wilkins; 2007.

[24] Lehman LH, Saeed M, Talmor D, Mark R and Malhotra A. Methods of Blood Pressure Measurement in the ICU. Critical Care Medicine 2013; 41(1): 34-40. doi:10.1097/CCM.0b013e318265ea46.

[25] Balzer F, Aronson S, Campagna JA, Ding L, Treskatsch S, Spies C and Sander M. High postoperative blood pressure after cardiac surgery is associated with acute kidney injury and death Journal of Cardiothoracic and Vascular Anesthesia 2016; http://dx.doi.org/10.1053/j.jvca.2016.05.040)

[26] Aronson S. Perioperative hypertensive emergencies. Current Hypertension Reports 2014; 16:448

[27] Lopes CT, Brunori EF, Cavalcante AM, Moorhead SA, Swanson E, Lopes JL and Barros ALBL. Factors associated with excessive bleeding after cardiac surgery: A prospective cohort study. Heart \& Lung 2016; 45: 64e69.

[28] Demirci C, Zemanb F, Schmidc C and Floerchingerc B. Early postoperative blood pressure and blood loss after cardiac surgery: A retrospective analysis. Intensive and Critical Care Nursing 2017. http://dx.doi.org/10.1016/j.iccn.2017.02.007.

[29] Nikolov NM, Fontes ML, White WD, Aronson S, Bar-Yosef S, Gaca JG, Podgoreanu MV, Stafford-Smith M, Newman MF and Mathew JP. Pulse pressure and long-term survival after coronary artery bypass graft surgery. Anesthesia and analgesia 2010; 110:335-40.

[30] Mangano DT. Biventricular function after myocardial revascularization in humans: Deterioration and recovery patterns during the first 24 hours. Anesthesiology 62:571-577, 1985

[31] Breisblatt WM, Stein KL, Wolfe CJ, Follansbee WP, Capozzi J, Armitage JM and Hardesty RL. Acute myocardial dysfunction and recovery: A common occurrence after coronary bypass surgery. Journal of the American College of Cardiology 1990; 15:1261-9.

[32] Ochagavía A, Baigorri F, Mesquida J, Ayuela JM, Ferrándiz A, García X, Monge MI, Mateu L, Sabatier C, Clau-Terré F, Vicho R, Zapata L, Maynar J and Gild A. Hemodynamic monitoring in the critically patient. Recommendations of the Cardiological Intensive Care and CPR Working Group of the Spanish Society of Intensive Care and Coronary Units. Medicina Intensiva 2014; 38(3):15469.

[33] Andriessen P, Schraa O, Van den Bosch-Ruis W, Harkel DJT, Settels JJ, Oetomo SB and Blanco CE. Feasibility of Noninvasive Continuous Finger Arterial Blood Pressure Measurements in Very Young Children, Aged 0-4 Years. Pediatric Research 2008; 63(6): 691-6.

[34] Gevers M, Hack WW, Ree EF, Lafeber HN and Westerhof N. Arterial blood pressure wave forms in radial and posterior tibial arteries in critically ill newborn infants. Journal of developmental physiology 1993; 19:179-85.

[35] Hofhuizen CM, Lemson J, Hemelaar AEA, Settels J, Schraa O, Singh SK, J. G. van der Hoeven JG and Scheffer GJ. Continuous non-invasive finger arterial pressure monitoring reflects intra-arterial pressure changes in children undergoing cardiac surgery. British Journal of Anaesthesia 2010; 105 (4): 493-500.

[36] Hori D, Max L, Laflam A, Brown C, Neufeld KJ, Adachi H, Sciortino C, Conte JV, Cameron DE, HogueJr CW and Mandal K. Blood Pressure Deviations From Optimal Mean Arterial Pressure During Cardiac Surgery Measured With a Novel Monitor of Cerebral Blood Flow and Risk for Perioperative Delirium: A Pilot Study. Journal of Cardiothoracic and Vascular Anesthesia 2016; 30(3):606-12.

[37] Jagadeesh AM, Singh NG and Mahankali S. A comparison of a continuous noninvasive arterial pressure (CNAPTM) monitor with an invasive arterial blood pressure monitor in the cardiac surgical ICU. Annals of Cardiac Anaesthesia 2012; 15:3.

[38] Heard SO, Lisbon A, Toth I and Ramasubramanian R. An evaluation of a new continuous blood pressure monitoring system in critically ill patients. The Journal of Clinical Anesthesia 2000; 12: 509-18. 
[39] Acierno MJ, Seaton D, Mitchell MA and da Cunha A. Agreement between directly measured blood pressure and pressures obtained with three veterinary specific oscillometric units in cats. Journal of the American Veterinary Medical Association 2010; 37: 402-6.

[40] Shih A, Robertson S, Vigani A, da Cunha A, Pablo L and Bandt C. Evaluation of an indirect oscillometric blood pressure monitor in normotensive and hypotensive anesthetized dogs. Journal of Veterinary Emergency and Critical Care 2010; 20 : 313-8.

[41] Almeida D, Barletta M, Mathews L, Graham L and Quandt J. Comparison between invasive blood pressure and a non-invasive blood pressure monitor in anesthetized sheep. Research in Veterinary Science 2014; 97: 582-6.

[42] Butterworth JF. Selecting an Inotrope for the Cardiac Surgery Patient. Journal of Cardiothoracic and Vascular Anesthesia 1993;7:(4):26-32.

[43] Butterworth JF, Prielipp RC, Royster RL, Spray BJ, Kon ND, Wallenhaupt LS and Zaloga GP. Dobutamine Increases Heart Rate More Than Epinephrine in Patients Recovering From Aortocoronary Bypass Surgery. Journal of Cardiothoracic and Vascular anesthesia 1992; 6(5): 535-41.

[44] DiSesa VJ, Brown E, Mudge GH, Collins JJ and Cohn LH. Hemodynamic comparison of dopamine and dobutamine in the postoperative volume-loaded, pressure-loaded, and normal ventricle. Journal of Thoracic Cardiovascular Surgery 1982; 83:256-63.

[45] Salomon NW, Plachetka JR and Copeland JG. Comparison of dopamine and dohutamine following coronary artery bypass grafting. The Annals of Thoracic Surgery 1982; 33:48-54.

[46] Wesseling KH. Finger arterial pressure measurement with Finapres. Zeitschrift Fur Kardiologie 1996; 85:38-44. 